\title{
B-cell CLL/lymphoma 3 promotes glioma cell proliferation and inhibits apoptosis through the oncogenic STAT3 pathway
}

\author{
JIANHENG WU ${ }^{1}$, LINFAN LI ${ }^{1}$, GUANGYUAN JIANG ${ }^{3}$, HUI ZHAN ${ }^{1}$ and NANNAN WANG ${ }^{2}$ \\ Departments of ${ }^{1}$ Neurosurgery, and ${ }^{2}$ Gastroenterology, The People's Hospital of Gaozhou, Gaozhou, \\ Guangdong 525200; ${ }^{3}$ Department of Neurosurgery, The Second People's Hospital of \\ Guangxi Zhuang Autonomous Region, Guilin 541000, P.R. China
}

Received July 26, 2016; Accepted September 21, 2016

DOI: $10.3892 /$ ijo.2016.3729

\begin{abstract}
Aberrant expression of oncogenes and/or tumor suppressors play fundamental roles in the pathogenesis of glioma. B-cell CLL/lymphoma 3 (BCL3) was previously found to be a putative proto-oncogene in human cancers and the decoy receptor DcR1 is induced in a p50/Bcl3-dependent manner and attenuates the efficacy of temozolomide in glioblastoma cells. However, its expression status, clinical significance and biological functions in glioma remain largely unknown. In the present study, the levels of BCL3 were overexpressed in glioma compared to normal brain tissues. Furthermore, high expression of BCL3 protein was confirmed by immunoblotting in glioma cells as compared with normal human astrocyte cell line. The positive expression of BCL3 was correlated with adverse prognostic features and reduced overall survival rate of glioma patients. BCL3 silencing resulted in prominent decreased proliferation, cell cycle arrest in G1 phase and increased apoptosis in U251 cells. In contrast, BCL3 overexpression in U87 cells remarkably facilitated proliferative ability and cell cycle progression and induced apoptosis. In vivo studies showed that BCL3 knockdown inhibited the tumor growth of U251 cells in a mouse xenograft model. Mechanistically, BCL3 positively regulated the abundance of STAT3, p-STAT3 and the downstream targets of STAT3 pathway including BCL2, MCL-1 and cyclin D1 in glioma cells. Furthermore, a positive correlation between BCL3 and STAT3 expression was observed in glioma specimens. Notably, we confirmed that STAT3 knockdown abolished the oncogenic roles of BCL3 in glioma. In conclusion, we suggest that BCL3 serves as an oncogene in glioma by modulating proliferation, cell cycle progression and apoptosis, and its oncogenic effects are mediated by the STAT3 signaling pathway.
\end{abstract}

Correspondence to: Professor Linfan Li, Department of Neurosurgery, The People's Hospital of Gaozhou, 89 Xiguan Road, Gaozhou, Guangdong 525200, P.R. China

E-mail: 1lf_guilin@163.com

Key words: BCL3, glioma, cell proliferation, cell cycle, apoptosis, STAT3

\section{Introduction}

Numerous studies have confirmed that oncogenes and/or tumor suppressors act as critical regulators for various cellular processes $(1,2)$, including the cell proliferation, apoptosis, differentiation and movements. Moreover, emerging studies have demonstrated that dysregulated oncogenes and/or tumor suppressors could modulate numerous oncogenic and tumor suppressive pathways in cancer cells and were actively implicated in the initiation of human cancers including glioma (3-5). In addition, oncogenes and/or tumor suppressors have been regarded as attractive therapeutic targets and promising biomarkers for human malignancies (6-8).

B-cell CLL/lymphoma 3 (BCL-3) is an atypical member of the $\mathrm{I} \kappa \mathrm{B}$ family (9) and can bind $\mathrm{NF}-\kappa \mathrm{B}$ homodimeric complexes of $\mathrm{p} 50$ or $\mathrm{p} 52$, which switches the transcriptional properties of the homodimers from a repressive to an activating state (10). The expression of mRNA and protein of BCL-3 has been reported to be overexpressed in breast cancer $(10,11)$, nasopharyngeal carcinoma (12), endometrial cancer (13), hepatocellular carcinoma (14) and colorectal cancer (15). Functionally, BCL-3 was found to regulate the colony formation and cell cycle progression by regulating ubiquitination-mediated degradation of c-Myc in colorectal cancer (16). Recently, Tu et al (14) reported that BCL3 promotes the tumor growth of hepatocellular carcinoma by regulating cell proliferation and the cell cycle through cyclin D1. A previous study demonstrated that STAT3, an important oncogene in human cancer, was a bona fide target of BCL3 in cervical cancer cell line (17). Recently, Mansour et al (18) reported that the decoy receptor DcR1 was induced in a p50/Bcl3-dependent manner and attenuated the efficacy of temozolomide in glioblastoma cells. However, the status of BCL3 and its exact roles in glioma have not been investigated.

In the present study, BCL3 was found to be overexpressed in glioma specimens and cells. The positive expression of BCL3 conferred adverse clinical parameters and reduced overall survival of the glioma patients. Moreover, BCL3 promoted the glioma cell proliferation and cell cycle progression and inhibited apoptosis. Interestingly, STAT3 pathway was recognized as a direct functional mediator of BCL3 in glioma. 
Table I. Correlation between the clinicopathological characteristics and BCL3 expression in gliomas.

\begin{tabular}{|c|c|c|c|c|}
\hline \multirow[b]{2}{*}{ Characteristics } & \multirow{2}{*}{$\begin{array}{l}\text { Total no. } \\
\text { of patients } \\
(\mathrm{n}=86)\end{array}$} & \multicolumn{2}{|c|}{ No. of patients } & \multirow[b]{2}{*}{ P-value } \\
\hline & & $\begin{array}{c}\text { BCL3 } \\
\text { positive }\end{array}$ & $\begin{array}{c}\text { BCL3 } \\
\text { negative }\end{array}$ & \\
\hline \multicolumn{5}{|l|}{ Age (years) } \\
\hline$<50$ & 42 & 20 & 22 & 0.286 \\
\hline$\geq 50$ & 44 & 26 & 18 & \\
\hline \multicolumn{5}{|l|}{ Gender } \\
\hline Male & 49 & 25 & 24 & 0.597 \\
\hline Female & 37 & 21 & 16 & \\
\hline \multicolumn{5}{|l|}{ Tumor size $(\mathrm{cm})$} \\
\hline$<5$ & 31 & 11 & 20 & $0.012^{\mathrm{a}}$ \\
\hline$\geq 5$ & 55 & 35 & 20 & \\
\hline \multicolumn{5}{|l|}{ KPS score } \\
\hline$<80$ & 51 & 22 & 29 & $0.020^{\mathrm{a}}$ \\
\hline$\geq 80$ & 35 & 24 & 11 & \\
\hline \multicolumn{5}{|l|}{ WHO grade } \\
\hline I+II & 28 & 17 & 21 & $0.029^{\mathrm{a}}$ \\
\hline III+IV & 58 & 39 & 19 & \\
\hline
\end{tabular}

WHO, World Health Organization; KPS, Karnofsky performance

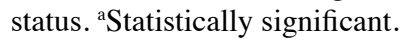

\section{Materials and methods}

Human specimens and cell culture. A total of 86 human glioma specimens and 20 normal brain tissues were collected from the People's Hospital of Gaozhou. Diagnoses were confirmed by pathologist. None of the patients received preoperative immunotherapy, chemotherapy or radiotherapy. These clinical samples were immediately fixed with formalin and embedded with paraffin after surgical resection. The written informed consents were obtained from every patient included in this study. The clinical stage was based on World Health Orgnization (WHO) grade (19). The demographic and clinical information of patients are shown in Table I. The protocols involving clinical tissues in the present study were approved by the Ethics Review Committee of the People's Hospital of Gaozhou.

The normal human astrocyte (NHA) cell line was obtained from the American Type Culture Collection (ATCC; Manassas, VA, USA). The human glioma cell lines (U87, T98, A172 and U251) were purchased from the Chinese Academy of Sciences (Shanghai, China). All cells were cultured in Dulbecco's modified Eagle's medium (DMEM; HyClone Laboratories, Logan, UT, USA) along with fetal bovine serum (10\%) (FBS; HyClone Laboratories), penicillin (100 U/ml) and streptomycin $(100 \mu \mathrm{g} / \mathrm{ml})$. Cell cultures were kept in an incubator containing $5 \% \mathrm{CO}_{2}$ and humidified atmosphere at $37^{\circ} \mathrm{C}$.

Immunohistochemistry (IHC). Before IHC staining, glioma specimens and normal brain tissues were fixed with formalin and embedded with paraffin. Then, the embedded tissues were cut into $4 \mu \mathrm{m}$ thick sections. IHC staining followed standard protocol to evaluate the expression level of BCL3 (sc-185; Santa Cruz Biotechnology, Santa Cruz, CA, USA) and STAT3 (\#9139; Cell Signaling Technology, Boston, MA, USA) in human tissues. The percentage of positive tumor cells was graded as per the following criteria: $0,<10 \% ; 1,10-30 \% ; 2$, $31-50 \%$; and $3,>50 \%$.

Cell transfection. BCL3 shRNA (sc-29789-SH) and corresponding non-target (NT) shRNA (sc-108060) were obtained from Santa Cruz Biotechnology. shRNAs transfection was performed using plasmid transfection reagent (sc-108061; Santa Cruz Biotechnology). Retroviral vector pMMP-BCL3 and pMMP-STAT3 were generated by inserting the cDNA into pMMP. Retrovirus packaging and transduction were previously described (20). A STAT3 specific siRNA and scrambled siRNA was obtained from OriGene Technologies, Inc. (Shanghai, China). The siRNAs were transduced into glioma cells with Lipofectamine 2000 (Invitrogen, Carlsbad, CA, USA) following the manufacturer's protocol.

$C C K-8$ and BrdU incorporation assay. Glioma cells that were treated with corresponding vectors were seeded into 96-well plates ( $1.5 \times 10^{3}$ cells/well); 24, 48, 72 and $96 \mathrm{~h}$ after transfection, the cell proliferation assay was performed by addition of $10 \mu 1$ Cell Counting kit-8 (CCK-8) solution (Beyotime Institute of Biotechnology, Shanghai, China) to each well, followed by incubation at $37^{\circ} \mathrm{C}$ for $2 \mathrm{~h}$. Absorbance was measured at a wavelength of $490 \mathrm{~nm}$ using a microplate reader (FlexStation III ROMV2.1.28; Molecular Devices, Sunnyvale, CA, USA). For 5-bromodeoxyuridine (BrdU) assays, $48 \mathrm{~h}$ after transfection, glioma cells grown on coverslips were incubated with $\mathrm{BrdU}$ at room temperature for $60 \mathrm{~min}$ and subsequently were incubated with the antibody of BrdU (Sigma-Aldrich, St. Louis, MO, USA) following the manufacturer's protocol.

Cell cycle assay. Forty-eight hours after transfection, glioma cells were collected with trypsin and fixed overnight at $4^{\circ} \mathrm{C}$ using $80 \%$ ethanol. Glioma cells were then incubated with propidium iodide (PI; Sigma-Aldrich) for $20 \mathrm{~min}$. Then, flow cytometry assays for glioma cells were performed with a FACSCalibur (BD Biosciences, Bedford, MA, USA).

Cell apoptosis assay. Apoptosis of cells was evaluated with the Annexin-V-FLUOS staining kit (Roche Diagnostics, Indianapolis, IN, USA) following standard protocol provided by the manufacturer. Then flow cytometry assay was performed with a FACSCalibur (BD Biosciences).

Western blot analysis. Total proteins were collected with RIPA lysis buffer, and $40 \mu \mathrm{g}$ protein were subjected to $4-20 \%$ SDS gel electrophoresis and were then transferred to PVDF membranes. Then, $5 \%$ milk blocked membranes were incubated with BCL3 (Santa Cruz Biotechnology), STAT3 (Cell Signaling Technology), p-STAT3 (Cell Signaling Technology), BCL2 (Cell Signaling Technology), MCL-1 (Cell Signaling Technology) or cyclin D1 (Cell Signaling Technology) antibodies, respectively, and subsequently incubated with matched secondary antibodies (Cell Signaling Technology). 


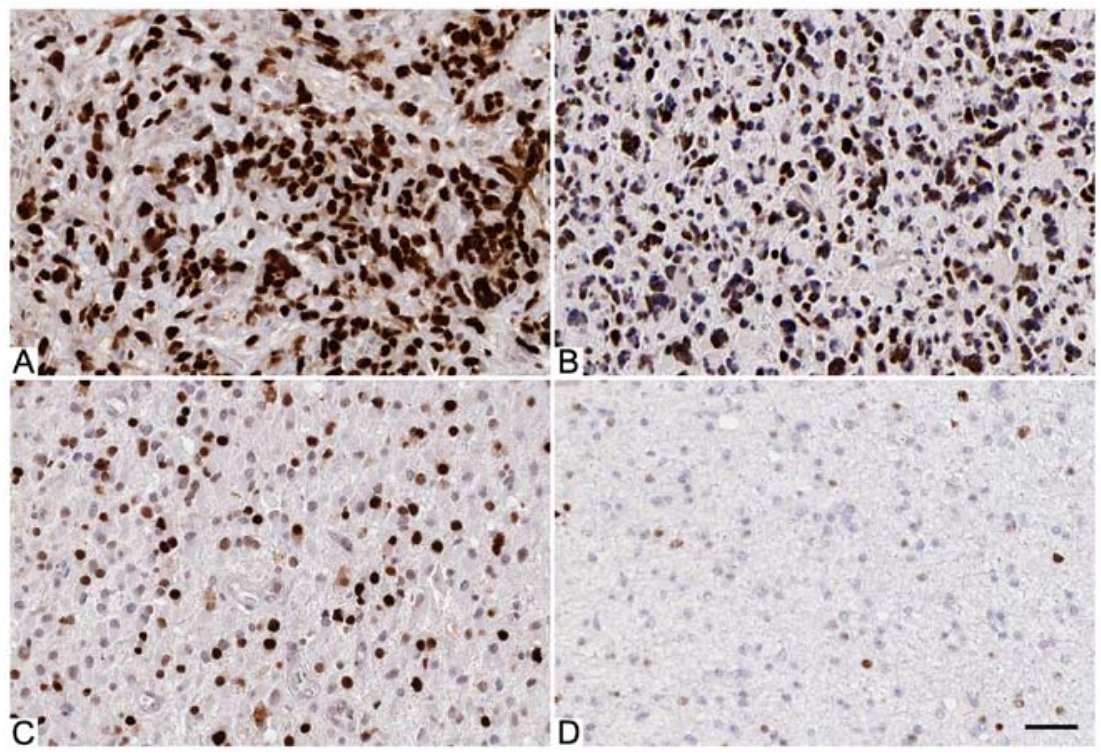

Figure 1. Representative immunostaining of BCL3 in glioma specimens. (A) High (IHC score, 3), (B) medium (IHC score, 2), (C) low (IHC score, 1) and (D) negative (IHC score, 0) expression of BCL-3 in glioma tissues. Scale bar, $50 \mu \mathrm{m}$.
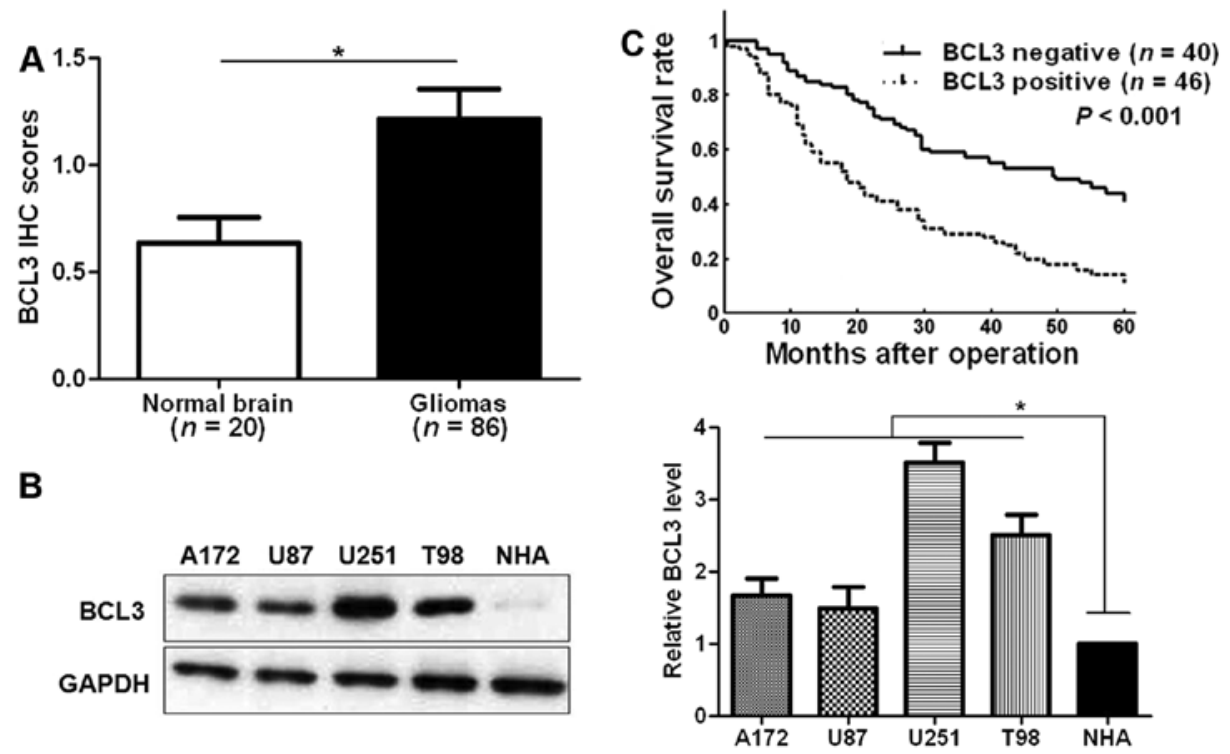

Figure 2. Prognostic significance of BCL3 in glioma. The expression differences of BCL3 between (A) glioma tissues and normal brain tissues, (B) 4 different glioma cell lines (A172, U87, U251 and T98 cells) and a normal human astrocyte (NHA) cell line. $\mathrm{n}=3$, ${ }^{*} \mathrm{P}<0.05$. (C) Compared with those with negative expression of BCL3, patients with positive expression of BCL3 had a significantly shorter overall survival.

Then, signals for each protein expression were detected with the Bio-Rad Gel imaging system. GAPDH (Cell Signaling Technology) was used as a loading control.

In vivo experiments. The subcutaneous tumor formation experiments were performed on nude mice. U251 cells $\left(5 \times 10^{6}\right)$ with BCL3 knockdown or cells of control group were suspended in PBS and were implanted into the back of mice via subcutaneous injection. Tumor volume was measured with calipers every 3 days. The xenograft tumor tissues were subjected to immunoblotting for Ki-67 (Cell Signaling Technology) and caspase-3 (Cell Signaling Technology). The protocols of in vivo experiments were approved by the Institutional Animal Care and Use Committee of the People's Hospital of Gaozhou.
Statistical analysis. All data were collected and showed as mean \pm standard error (SE). GraphPad Prism 5 software (GraphPad Software, Inc., San Diego, CA, USA) was used in the present study to perform statistical analysis. $\mathrm{P}<0.05$ was considered to be statistically different.

\section{Results}

Overexpression of BCL3 in glioma tissues and cells confers disease progression. IHC was initially performed to detect BCL3 expression in glioma specimens and normal brain tissues. IHC sections were scored according to the percentage of positive cells (Fig. 1). The level of BCL3 was significantly upregulated in glioma tissues as compared with normal brain 
A
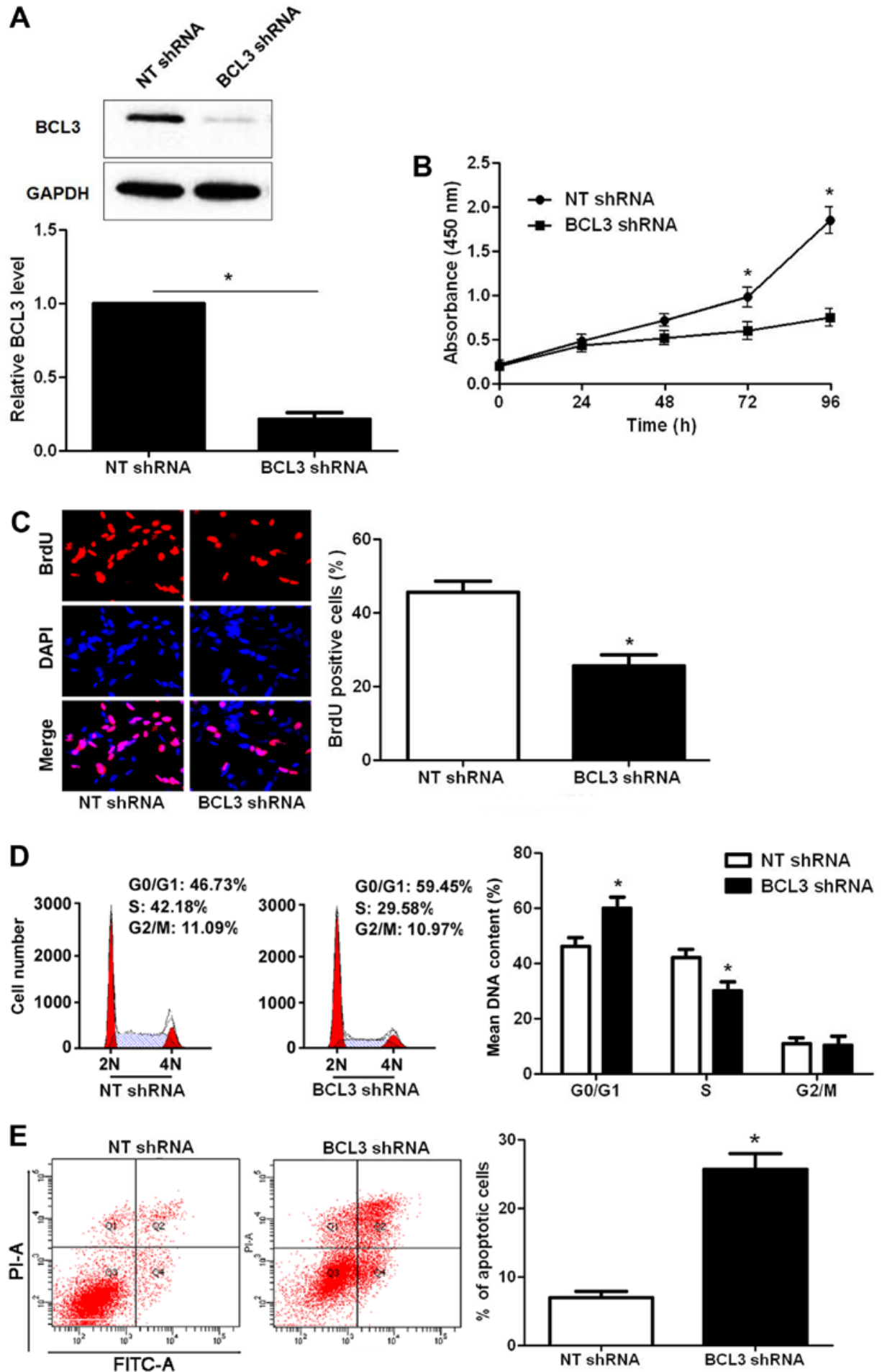

Figure 3. BCL3 knockdown results in decreased cell proliferation, cell cycle arrest and apoptosis in U251 cells. (A) U251 cells that were transfected with NT shRNA or BCL3 shRNA were subjected to immunoblotting for BCL3. $n=3$, ${ }^{*} \mathrm{P}<0.05$. (B) CCK-8 assays indicated that the proliferative ability of U251 cells was reduced after BCL3 knockdown. $\mathrm{n}=3$, ${ }^{\mathrm{P}} \mathrm{P}<0.05$. (C) BrdU assays confirmed that BCL3 silencing inhibited U251 cell proliferation. $\mathrm{n}=3$, ${ }^{\prime} \mathrm{P}<0.05$. (D) Cell cycle progression was significantly suppressed after BCL3 knockdown with G1-phase arrest in U251 cells. $\mathrm{n}=3,{ }^{*} \mathrm{P}<0.05$. (E) BCL3 knockdown led to an obviously increased percentage of apoptotic U251 cells. $n=3,{ }^{*} \mathrm{P}<0.05$.

tissues $(\mathrm{P}<0.05$; Fig. 2A). Furthermore, our data revealed that BCL3 expression in four glioma cell lines (A172, U87, U251 and T98 cells) was evidently increased compared to an NHA cell line ( $\mathrm{P}<0.05$, respectively; Fig. $2 \mathrm{~B})$. BCL3 staining was considered as positive expression in 46 cases of glioma according to IHC scores (1-3). As shown in Table I, positive expression of BCL3 in glioma patients was positively correlated with large tumor size $(\mathrm{P}=0.012)$, high Karnofsky performance status (KPS) score $(\mathrm{P}=0.020)$ and advanced WHO grade $(\mathrm{P}=0.029)$. Then, we examined the prediction value of BCL3 for the prognosis of glioma patients. Compared with those with negative expression of BCL3, patients that showed positive expression of BCL3 had significant reduced 5 -year overall survival $(\mathrm{P}<0.001 ;$ Fig. $2 \mathrm{C})$. The above indicate 

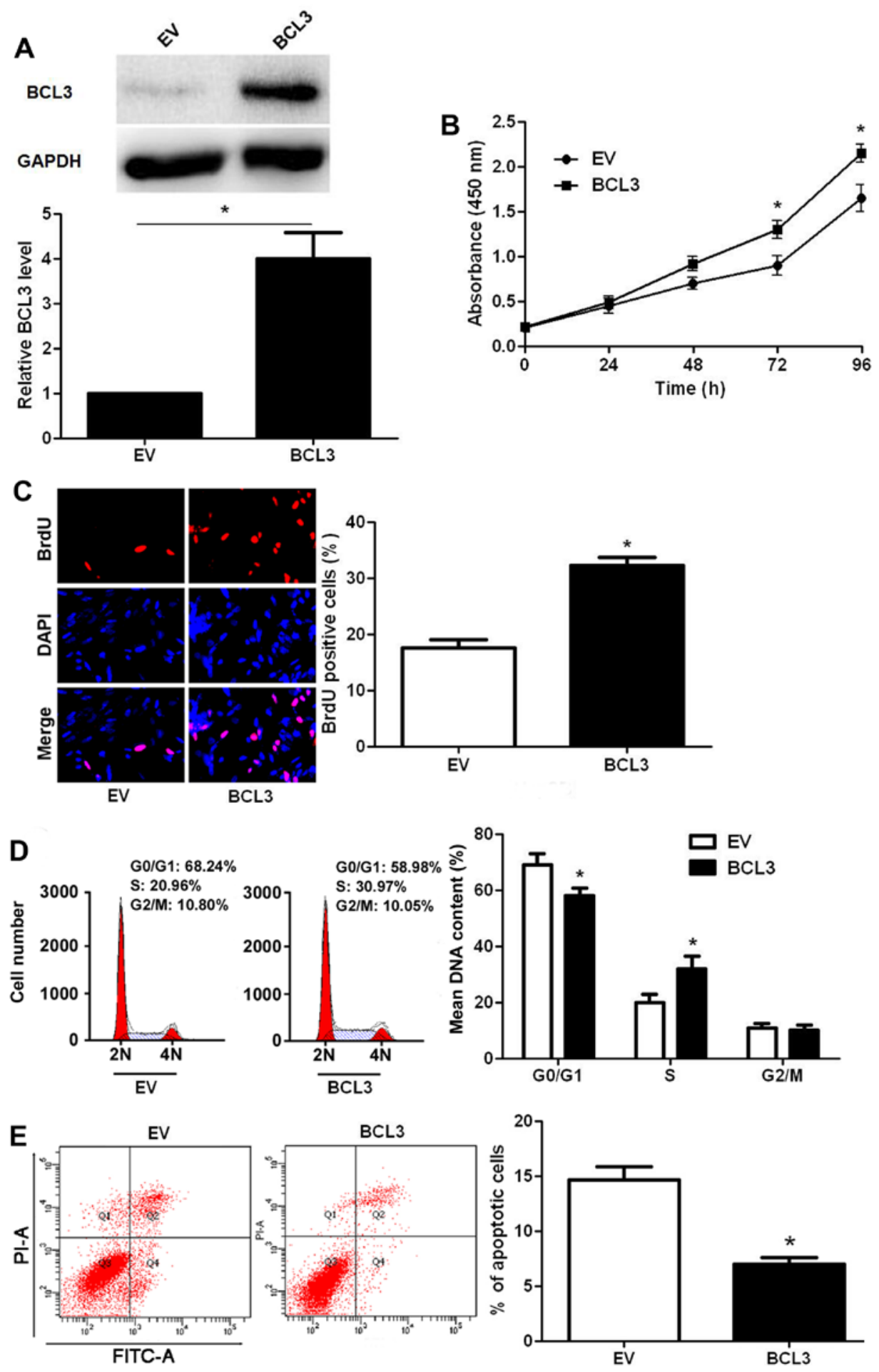

Figure 4. BCL3 overexpression facilitates proliferation, cell cycle progression and anti-apoptosis in U87 cells. (A) U87 cells that were infected with empty vector (EV) or BCL3 retroviruses were subjected to immunoblotting for BCL3. $n=3,{ }^{*} \mathrm{P}<0.05$. (B) CCK-8 assays indicated that the proliferative ability of U87 cells was enhanced after BCL3 overexpression. $n=3,{ }^{*} P<0.05$. (C) BrdU assays confirmed that BCL3 overexpression promoted U87 cell proliferation. $n=3$, ${ }^{*} \mathrm{P}<0.05$. (D) BCL3 knockdown facilitated cell cycle progression in U87 cells. $n=3,{ }^{*} \mathrm{P}<0.05$. (E) BCL3 knockdown led to a significant decreased percentage of apoptotic U87 cells. $n=3,{ }^{*} \mathrm{P}<0.05$.

that BCL3 can potentially act as biomarker and prognostic indicator for glioma.

BCL3 regulates biological function in glioma cell lines. Next, we disclosed the exact roles of BCL3 in glioma cells. Since increased proliferation, aberrant cell cycle progression and reduced apoptosis are well-recognized hallmarks of human cancer (21), we examined whether BCL3 regulated these biological processes of glioma cells. BCL3 knockdown was confirmed by immunoblotting after BCL3 shRNA transfection $(\mathrm{P}<0.05$; Fig. 3A). CCK- 8 and BrdU assays indicated that BCL3 knockdown significantly reduced the proliferative ability of $\mathrm{U} 251$ cells ( $\mathrm{P}<0.05$, respectively; Fig. 3B and $\mathrm{C}$ ). Moreover, BCL3 knockdown led to a prominent cell cycle 

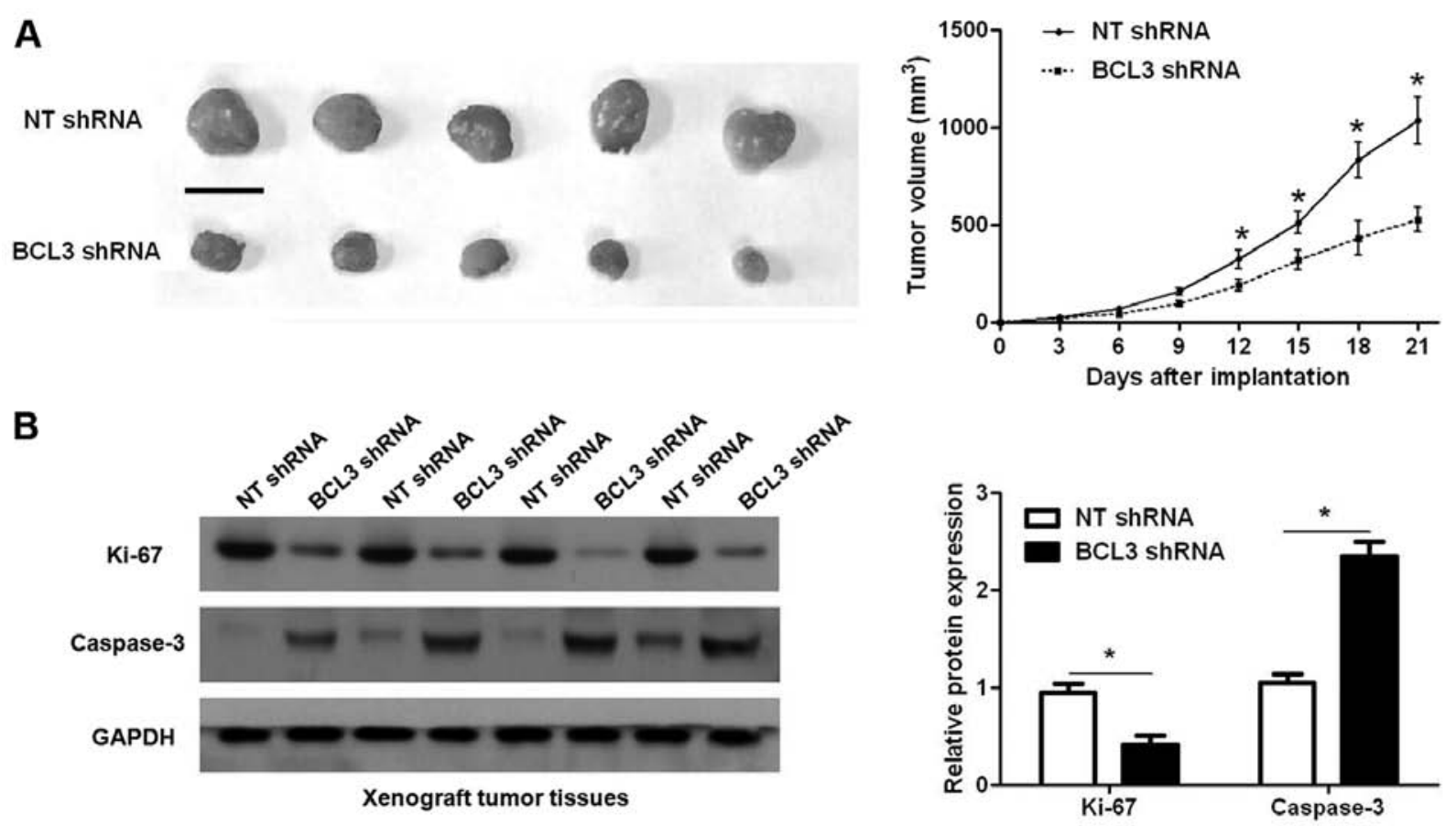

Figure 5. BCL3 knockdown slows down tumor growth in vivo. (A) U251 cells that were transfected with NT shRNA and BCL3 shRNA, respectively, were implanted into nude mice via subcutaneous injection. The tumor growth curves indicated that BCL3 knockdown significantly suppressed the tumor growth of glioma in vivo. $\mathrm{n}=5,{ }^{*} \mathrm{P}<0.05$. (B) The xenograft tumor tissues were subjected to western blotting for Ki-67 and caspase-3. BCL3 knockdown resulted in prominent decrease of Ki-67 level and increase of caspase- 3 level. $n=5,{ }^{*} \mathrm{P}<0.05$.

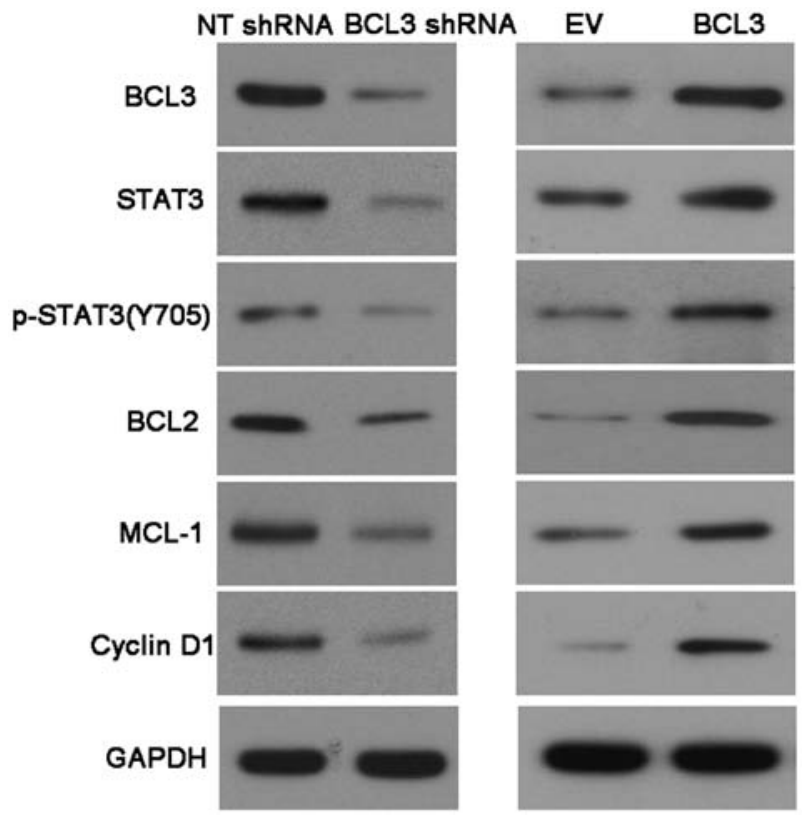

Figure 6. BCL3 regulates the oncogenic STAT3 pathway in glioma cells. U87 and $\mathrm{U} 251$ cells that were transfected with corresponding vectors were subjected to immunoblotting for BCL3, STAT3, p-STAT3, BCL2, MCL-1 and cyclin D1. BCL3 positively regulated the expression of STAT3 and p-STAT3, and coordinately modulated the downstream molecules of STAT3 pathway including BCL1, MCL-1 and cyclin D1.

arrest at G1-phase $(\mathrm{P}<0.05$; Fig. 3D). The portion of apoptotic cells were evidently increased after BCL3 silencing ( $\mathrm{P}<0.05$; Fig. 3E). On the contrary, infection of BCL3 retroviruses led to a significantly increased expression of BCL3 in U87 cells
(P<0.05; Fig. 4A). Functionally, BCL3 overexpression facilitated cell proliferation $(\mathrm{P}<0.05$, respectively; Fig. $4 \mathrm{~B}$ and $\mathrm{C})$ and cell cycle progression $(\mathrm{P}<0.05 ;$ Fig. $4 \mathrm{D})$, while suppressed apoptosis in U87 cells $(\mathrm{P}<0.05$; Fig. 4E). Furthermore, we used a subcutaneous tumor formation model to investigate whether BCL3 could affect the growth of glioma cells in a mouse xenograft model. As shown in Fig. 5A, BCL3 knockdown significantly reduced the tumor growth of U251 cells in mice $(\mathrm{P}<0.05)$. Furthermore, immunoblotting of Ki-67 and caspase- 3 indicated BCL3 knockdown inhibited proliferation and induced apoptosis of glioma cells in vivo $(\mathrm{P}<0.05$, respectively; Fig. 5B). Thus, BCL3 functions as an oncogene by promoting proliferation and the process of cell cycle, and inhibiting apoptosis in glioma.

BCL3 positively regulates STAT3 abundance in glioma. A previous study demonstrated that STAT3, an important oncogene in human cancer, was a bona fide target of BCL3 in cervical cancer cell line (17). U251 cells that were transduced with NT shRNA and BCL3 shRNA, respectively, were subjected to immunoblotting for BCL3, STAT3 and p-STAT3 expression. As expected, BCL3 knockdown significantly reduced STAT3 and p-STAT3 expression in vitro (Fig. 6). Since BCL2, MCL-1 and cyclin D1 are downstream molecules involved in STAT3 signaling pathway (22). Notably, BCL3 knockdown coordinately reduced the levels of BCL2, MCL-1 and cyclin D1 in U251 cells (Fig. 6). In contrast, BCL3 overexpression resulted in an obvious increase of STAT3, p-STAT3, BCL2, MCL-1 and cyclin D1 protein expression (Fig. 6) in U87 cells. Furthermore, IHC was performed to detect the expression of STAT3 in glioma specimens. Interestingly, Spearman's correlation analysis indicated that BCL3 was 


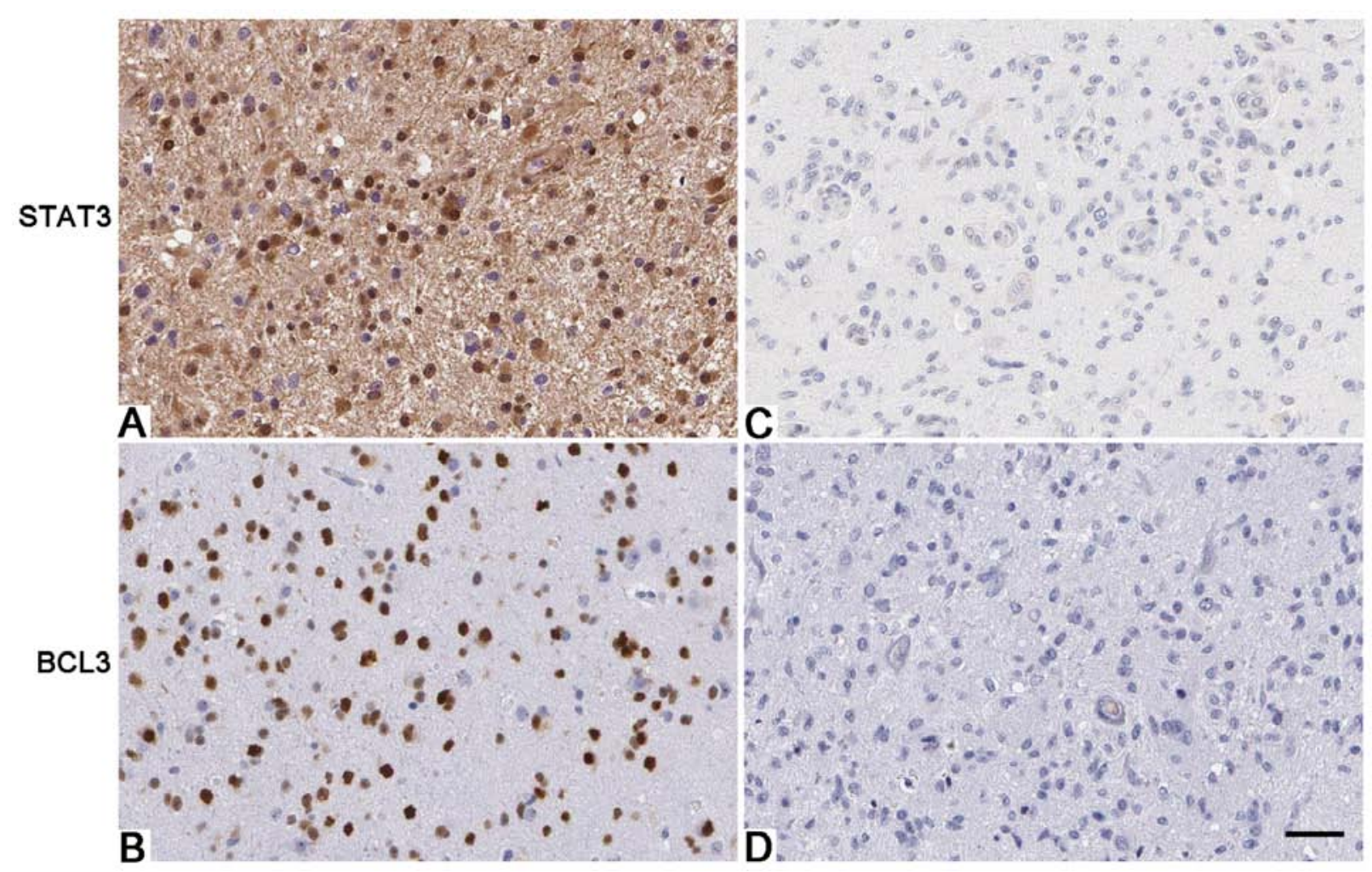

Figure 7. BCL3 positively correlates with STAT3 in glioma specimens. Representative immunohistochemical staining showed strong staining of STAT3 (A) in BCL3 positive-expressing tumor (B) and weak staining of STAT3 (C) in BCL3 negative-expressing tumor (D). Scale bar, $50 \mu \mathrm{m}$.

positively correlated with STAT3 expression in glioma specimens ( $\mathrm{r}=0.673, \mathrm{P}<0.001$; Fig. 7).

STAT3 knockdown reverses the oncogenic role of BCL3 in vitro. To determine whether STAT3 silencing could attenuate the oncogene function of BCL3 in glioma, a series of rescue experiments were performed. STAT3 siRNA were employed for STAT3 knockdown in BCL3 overexpressing U87 cells $(\mathrm{P}<0.05$; Fig. 8A). Notably, STAT3 knockdown abolished the oncogenic effects of BCL3 overexpression on U87 cells with decreased cell proliferation $(\mathrm{P}<0.05$, respectively; Fig. $8 \mathrm{~B}$ and $\mathrm{C})$, cell cycle arrest $(\mathrm{P}<0.05$; Fig. 8D) and increased apoptosis $(\mathrm{P}<0.05$; Fig. 8E). Our data indicate that STAT3 mediates the functions of BCL3 in glioma cells.

\section{Discussion}

Increasing evidence confirms that oncogenes play critical roles in the progression of human malignancies including glioma (23). Functionally, oncogenes were found to modulate the proliferation, apoptosis and metastasis of glioma cells (24). Moreover, oncogenes were proposed as novel biomarkers and treatment targets for glioma (25). Therefore, elucidating the expression level, clinical significance and biological function of specific oncogene in glioma will greatly contribute to the diagnosis and treatment of glioma. Here, overexpression of BCL3 was observed in glioma specimens and cells. In addition, positive expression of BCL3 in glioma was directly correlated with the adverse clinical features. Importantly, we suggest that positive expression of BCL3 was associated with the reduced 5-year overall survival of glioma patients.
Therefore, these data in the present study demonstrate that BCL3 can act as a promising biomarker and prognostic indicator for glioma.

BCL3, a well-known proto-oncogene, is deregulated in solid malignancies and exerts an oncogenic role by regulating proliferation and cell death (17). Study of breast cancer confirmed that BCL3-dependent stabilization of CtBP1 was crucial for the inhibition of apoptosis and tumor progression (11). Moreover, BCL3 promoted the cell proliferation of ovarian cancer cells (26). In this study, in vitro experiments confirmed that BCL3 promoted the proliferative ability and cell cycle progression of glioma cells while induced apoptosis. Moreover, in vivo experiments showed that BCL3 knockdown could inhibit the tumor growth of glioma in nude mice. Therefore, this study confirms that BCL3 exerts an oncogenic role by modulating proliferation, cell cycle progression and apoptosis in glioma.

STAT3, an important oncogene, was found to be overexpressed and persistently active in $\sim 70 \%$ of human cancers including glioma (27-30). STAT3 activation is implicated in cell proliferation, apoptosis, migration, invasion and angiogenesis in glioma (30). Phosphorylation of tyrosine residue (Y705) is necessary for STAT3 activation and positively regulates its downstream targets including BCL2, MCL-1 and cyclin D1. Cyclin D1 knockdown confers cell cycle arrest at G1-phase (14). Moreover, p-STAT3 knockdown facilitates apoptosis via downregulation of MCL-1 and BCL2 (31). Here, we presented that BCL3 positively regulated the levels of STAT3, p-STAT3 and its downstream molecules including BCL2, MCL-1 and cyclin D1. Furthermore, IHC staining indicated that BCL3 protein levels positively correlated with 
A
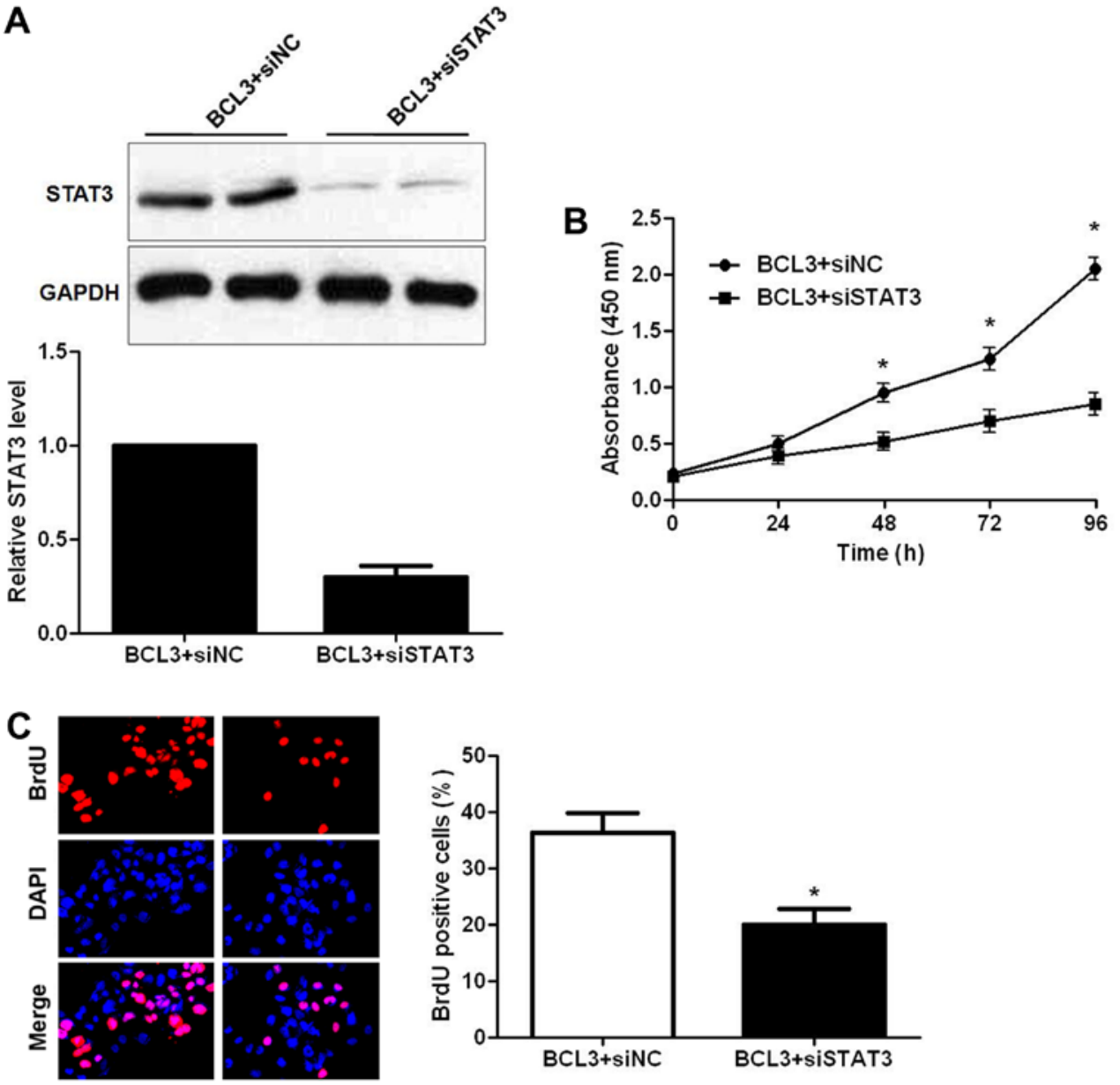

D
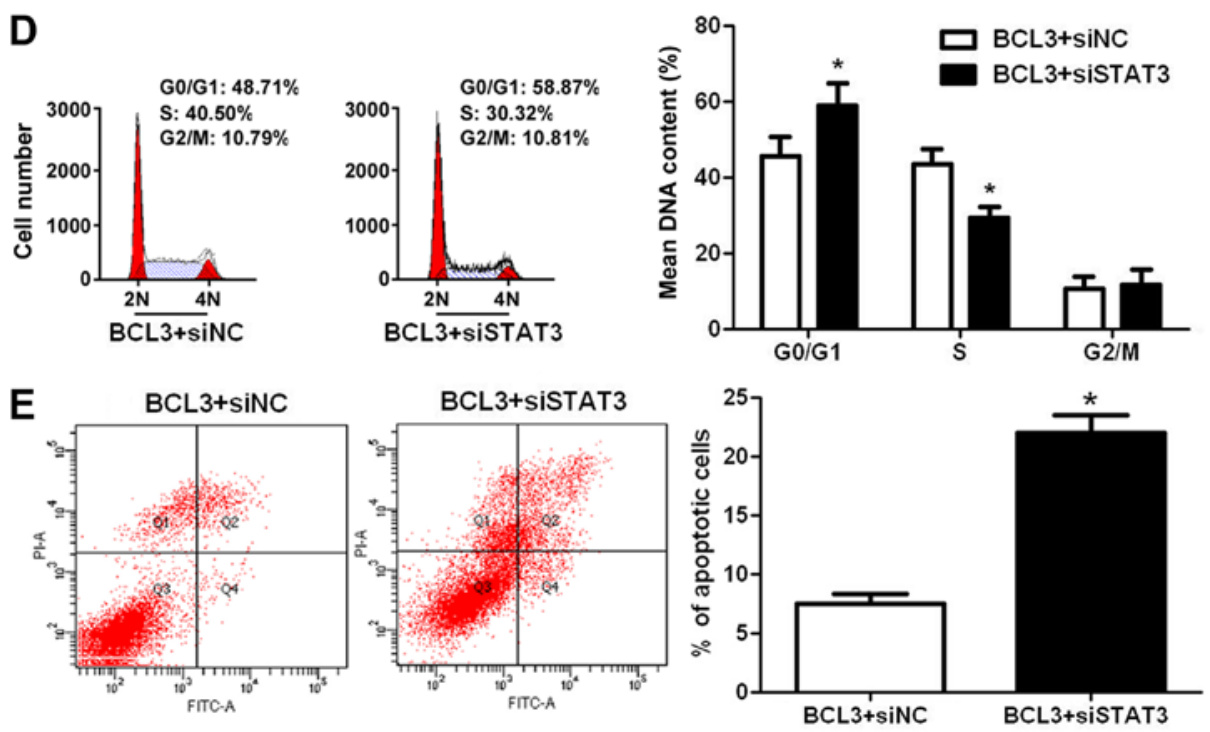

Figure 8. STAT3 knockdown reverses the oncogenic role of BCL3 in glioma cells. (A) Western blot analyses confirmed that STAT3 expression was knocked down by siRNA in BCL3 overexpressing U87 cells. $n=3$. (B) STAT3 knockdown evidently reduced proliferation in BCL3 overexpressing U87 cells. $n=3$, ${ }^{*} \mathrm{P}<0.05$. (C) The proliferative ability of BCL3 overexpressing U87 cells were prominently reduced after STAT3 knockdown. $n=3$, "P<0.05. (D) STAT3 knockdown led to G1-phase arrest in BCL3 overexpressing U87 cells. $n=3,{ }^{*} \mathrm{P}<0.05$. (E) STAT3 knockdown resulted in an obviously increased percentage of apoptotic cells in BCL3 overexpressing U87 cells. $n=3,{ }^{*} P<0.05$.

STAT3 protein expression in human glioma specimens. These data further demonstrate that STAT3 is a downstream target of BCL3 in glioma. Notably, STAT3 knockdown could abrogate the influence of BCL3 overexpression on proliferation, the process of cell cycle and apoptosis in glioma cells. Therefore, these results indicate that STAT3 serves as not only a downstream target of BCL3 but also a functional mediator of BCL3 in glioma.

Collectively, we present that BCL3 is overexpressed in glioma and its positive expression is correlated with poor 
clinical parameters and survival. BCL3 promotes the tumor growth of glioma through regulating proliferative ability, process of cell cycle and apoptosis. Mechanistically, this study finds that BCL3 functions as an oncogenic factor by activating STAT3 pathway. Taken together, BCL3 may serve as a clinical indicator and a drug target for glioma patients.

\section{Acknowledgements}

The authors thank all the patients who participated in the present study.

\section{References}

1. Viale A, Pettazzoni P, Lyssiotis CA, Ying H, Sánchez N, Marchesini M, Carugo A, Green T, Seth S, Giuliani V, et al: Oncogene ablation-resistant pancreatic cancer cells depend on mitochondrial function. Nature 514: 628-632, 2014.

2. Antal CE, Hudson AM, Kang E, Zanca C, Wirth C, Stephenson NL, Trotter EW, Gallegos LL, Miller CJ, Furnari FB, et al: Cancer-associated protein kinase $\mathrm{C}$ mutations reveal kinase's role as tumor suppressor. Cell 160: 489-502, 2015.

3. Chistiakov DA and Chekhonin VP: Extracellular vesicles shed by glioma cells: Pathogenic role and clinical value. Tumour Biol 35: 8425-8438, 2014

4. Zhang XQ and Leung GK: Long non-coding RNAs in glioma: Functional roles and clinical perspectives. Neurochem Int 77: 78-85, 2014.

5. Chautard E, Ouédraogo ZG, Biau J and Verrelle P: Role of Akt in human malignant glioma: From oncogenesis to tumor aggressiveness. J Neurooncol 117: 205-215, 2014.

6. Katsetos CD, Reginato MJ, Baas PW, D'Agostino L, Legido A, Tuszyn Ski JA, Dráberová E and Dráber P: Emerging microtubule targets in glioma therapy. Semin Pediatr Neurol 22: 49-72, 2015.

7. Awad AJ, Burns TC, Zhang Y and Abounader R: Targeting MET for glioma therapy. Neurosurg Focus 37: E10, 2014.

8. Huse JT and Aldape KD: The evolving role of molecular markers in the diagnosis and management of diffuse glioma. Clin Cancer Res 20: 5601-5611, 2014.

9. Bours V, Franzoso G, Azarenko V, Park S, Kanno T, Brown K and Siebenlist U: The oncoprotein Bcl-3 directly transactivates through kappa B motifs via association with DNA-binding p50B homodimers. Cell 72: 729-739, 1993.

10. Cogswell PC, Guttridge DC, Funkhouser WK and Baldwin AS Jr: Selective activation of NF-kappa B subunits in human breast cancer: Potential roles for NF-kappa B2/p52 and for Bcl-3. Oncogene 19: 1123-1131, 2000.

11. Choi HJ, Lee JM, Kim H, Nam HJ, Shin HJ, Kim D, Ko E, Noh DY, $\mathrm{Kim} \mathrm{KI}, \mathrm{Kim} \mathrm{JH}$, et al: Bcl3-dependent stabilization of CtBP1 is crucial for the inhibition of apoptosis and tumor progression in breast cancer. Biochem Biophys Res Commun 400: 396-402, 2010.

12. Thornburg NJ, Pathmanathan R and Raab-Traub N: Activation of nuclear factor-kappaB p50 homodimer/Bcl-3 complexes in nasopharyngeal carcinoma. Cancer Res 63: 8293-8301, 2003

13. Pallares J, Martínez-Guitarte JL, Dolcet X, Llobet D, Rue M, Palacios J, Prat J and Matias-Guiu X: Abnormalities in the NF-kappaB family and related proteins in endometrial carcinoma. J Pathol 204: 569-577, 2004.

14. Tu K, Liu Z, Yao B, Xue Y, Xu M, Dou C, Yin G and Wang J: BCL-3 promotes the tumor growth of hepatocellular carcinoma by regulating cell proliferation and the cell cycle through cyclin D1. Oncol Rep 35: 2382-2390, 2016.
15. Puvvada SD, Funkhouser WK, Greene K, Deal A, Chu H, Baldwin AS, Tepper JE and O'Neil BH: NF- $\kappa$ B and Bcl-3 activation are prognostic in metastatic colorectal cancer. Oncology 78: 181-188, 2010.

16. Liu Z, Jiang Y, Hou Y, Hu Y, Cao X, Tao Y, Xu C, Liu S, Wang S, Wang L, et al: The I $\kappa \mathrm{B}$ family member Bcl-3 stabilizes c-Myc in colorectal cancer. J Mol Cell Biol 5: 280-282, 2013.

17. Maldonado V, Espinosa M, Pruefer F, Patiño N, CeballosCanciono G, Urzua U, Juretic N and Melendez-Zajgla J: Gene regulation by BCL3 in a cervical cancer cell line. Folia Biol (Praha) 56: 183-193, 2010.

18. Mansour NM, Bernal GM, Wu L, Crawley CD, Cahill KE, Voce DJ, Balyasnikova IV, Zhang W, Spretz R, Nunez L, et al: Decoy receptor DcR1 is induced in a p50/Bcl3-dependent manner and attenuates the efficacy of temozolomide. Cancer Res 75: 2039-2048, 2015.

19. Pessôa IA, Sagica FE, Anselmo NP, Brito JR and de Oliveira EH: IDH1 and IDH2 mutations in different histologic subtypes and WHO grading gliomas in a sample from Northern Brazil. Genet Mol Res 14: 6533-6542, 2015.

20. Tu K, Li J, Verma VK, Liu C, Billadeau DD, Lamprecht G, Xiang X, Guo L, Dhanasekaran R, Roberts LR, et al: Vasodilatorstimulated phosphoprotein promotes activation of hepatic stellate cells by regulating Rab11-dependent plasma membrane targeting of transforming growth factor beta receptors. Hepatology 61 : 361-374, 2015.

21. Cipriano R, Miskimen KL, Bryson BL, Foy CR, Bartel CA and Jackson MW: Conserved oncogenic behavior of the FAM83 family regulates MAPK signaling in human cancer. Mol Cancer Res 12: 1156-1165, 2014.

22. Li H, Liu YW, Wang H, Zhou Q, Li JJ, Annie H, Qi ST and Lu YT: MiR-519a functions as a tumor suppressor in glioma by targeting the oncogenic STAT3 pathway. J Neurooncol 128: $35-45,2016$.

23. Hartel I, Ronellenfitsch M, Wanka C, Wolking S, Steinbach JP and Rieger J: Activation of AMP-activated kinase modulates sensitivity of glioma cells against epidermal growth factor receptor inhibition. Int J Oncol 49: 173-180, 2016.

24. Foster KA, Jane EP, Premkumar DR, Morales A and Pollack IF: NVP-BKM120 potentiates apoptosis in tumor necrosis factorrelated apoptosis-inducing ligand-resistant glioma cell lines via upregulation of Noxa and death receptor 5. Int J Oncol 47: 506-516, 2015.

25. Wang H, Wang Y, Bao Z, Zhang C, Liu Y, Cai J and Jiang C: Hypomethylated Rab27b is a progression-associated prognostic biomarker of glioma regulating MMP-9 to promote invasion. Oncol Rep 34: 1503-1509, 2015.

26. Guan Y, Yao H, Zheng Z, Qiu G and Sun K: MiR-125b targets BCL3 and suppresses ovarian cancer proliferation. Int J Cancer 128: 2274-2283, 2011

27. Wu ZL, Song YQ, Shi YF and Zhu J: High nuclear expression of STAT3 is associated with unfavorable prognosis in diffuse large B-cell lymphoma. J Hematol Oncol 4: 31, 2011.

28. Chen Y, Wang J, Wang X, Liu X, Li H, Lv Q, Zhu J, Wei B and Tang Y: STAT3, a poor survival predicator, is associated with lymph node metastasis from breast cancer. J Breast Cancer 16: 40-49, 2013.

29. Takemoto S, Ushijima K, Kawano K, Yamaguchi T, Terada A, Fujiyoshi N, Nishio S, Tsuda N, Ijichi M, Kakuma T, et al: Expression of activated signal transducer and activator of transcription-3 predicts poor prognosis in cervical squamous-cell carcinoma. Br J Cancer 101: 967-972, 2009.

30. Brantley EC and Benveniste EN: Signal transducer and activator of transcription-3: A molecular hub for signaling pathways in gliomas. Mol Cancer Res 6: 675-684, 2008.

31. Iwamaru A, Szymanski S, Iwado E, Aoki H, Yokoyama T, Fokt I, Hess K, Conrad C, Madden T, Sawaya R, et al: A novel inhibitor of the STAT3 pathway induces apoptosis in malignant glioma cells both in vitro and in vivo. Oncogene 26: 2435-2444, 2007. 